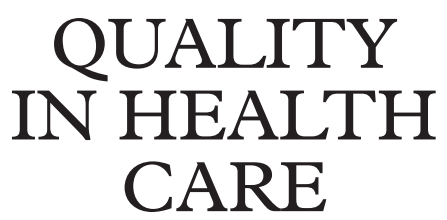

\title{
Editorials
}

\section{Multidisciplinary teamwork: the good, bad, and everything in between}

Teams make up the building blocks of health care and every team - from the executive to the coal face-is composed of different professionals, ideally possessing a variety of skills necessary to produce safe and effective care. ${ }^{1}$ We are constantly reminded of the value of diversity within teams, but the reality is that working together from a variety of perspectives is sometimes difficult to achieve. The paper by Jenkins et al in this issue of Quality in Health Care shows, for example, that, unless roles are well defined and understood, responsibility for giving different types of information to patients could easily result in overload to the patient, differences in messages, and gaps in certain areas.

The difficulties of multidisciplinary teamwork are also apparent in differing attitudes towards the way to bring about a good outcome, and even what actually constitutes a good outcome. For example, in teams caring for patients with stroke there are a number of "ideal outcomes" from the various stakeholders and staff involved-complete mobility is desired by the patient, physical immobility but good mental ability by the carer, compliance with the regime is required from some health workers, and (I have heard say) death from the contracts manager. Just as feedback from the cancer team audit would be helpful in producing better communication in the future, ${ }^{2}$ so discussing different approaches to care and differing ideas of best outcome will also keep the team on what is inevitably a wavy line along the best practice meridian.

What makes healthcare teams so different from those in other types of organisation is that team members have differing allegiances, not only to the team but also to their professional groups. For example, if a member of the nursing staff abuses a patient on a psychiatric ward, who has ultimate authority to deal with this - the nursing line manager or the consultant psychiatrist who is seen by some as the team leader? In commercial organisations such questions would be easily answered, but history and professionalism play their part in making questions of authority and responsibility in the health service team much more complex. ${ }^{3}$ Reinforcement of the supra-goalpatient safety-can help to push people beyond professional barriers, but clarity about authority and accountability in health care teams is long overdue.

There is another warning to heed against complacency in terms of teams: in these days of constant measurement and the drive towards reporting of poor care, ${ }^{4}$ we need to look beyond the data laid before us. A study by Edmondson $^{5}$ on medication errors in nursing teams showed that poor teams produced fewer errors; however, poor teams had authoritarian team leaders and so the likely explanation for this surprising finding is that data were being manipulated in poor teams and shown honestly in good open communicating teams. Clearly, teams need to be rewarded for their reporting systems and use of feedback rather than for the data alone.

These are not issues to dodge, but neither do they make good multidisciplinary teamwork an impossible task to achieve. Such teams are the reality that we work in and, although rarely captured in data, their diversity does actually help patient care. ${ }^{6}$ It is possible that this happens because of the increasing findings which show that members of good teams are significantly less stressed than others. ${ }^{7}$ Moreover, in a study of house officers, those who appreciated that they were part of a multidisciplinary team (as opposed to simply being bottom of a medical hierarchy) had far lower stress levels than those who did not, ${ }^{8}$ probably because they were able to learn from the diversity of skills that surrounded them and could look further than their medical colleagues for support. Since we know that lower stress means better patient care, ${ }^{9}$ it is also likely (as well as common sense) that better teams produce better care through having more cheerful staff, probably through their greater sense of participation and support. Somewhere in this equation lies the issue of sufficient resources-sufficient to allow time for good communication within the team and with the patient.

So multidisciplinary teams are likely to be better for everyone, but to keep them working well needs skill as well as recognition that this is always a long term task requiring constant attention and adjustment. Good team leaders are essential for maintaining patient safety ${ }^{1}$ and the sooner we get them the training and support they need for this task, the better the quality of care is likely to be.

J FIRTH-COZENS

Director, Centre for Clinical Psychology,

University of Northumbria at Newcastle,

Newcastle upon Tyne NE7 7AX, UK

jenny.firth-cozens@unn.ac.uk

1 Firth-Cozens J. Cultures for effective learning. In: Vincent C, ed. Clinical risk management. London: BMJ Books, 2001: 355-68.

2 Jenkins VA, Fallowfield LJ, Poole K. Are members of multidisciplinary teams in breast cancer aware of each others' informational roles? Quality in Health Care 2001;10:70-5.

3 Stokes J. Institutional chaos and personal stress. In: Obholzer A, Zagier Roberts $\mathrm{V}$, eds. The unconscious at work: individual and organizational stress in human services. London: Routledge, 1995: 121-8. 
4 Department of Health. An organisation with a memory: report of the expert group on learning from adverse events in the NHS. London: Department of

5 Edmondson AC. Learning from mistakes is easier said than done: group and organizational influences on the detection and correction of human error. $\mathcal{F}$ Appl Behav Sci 1996;32:5-28.

6 Adorian D, Silverberg DS, Tomer D, et al. Group discussions with the health care team: a method of improving care of hypertension in general practice. f Hum Hypertens 1990;4:265-8.
7 Carter AJ, West MA. Sharing the burden: team work in health care setting. In: Firth-Cozens J, Payne RL, eds. Stress in health professionals: psychological and organizational causes and interventions. Chichester: John Wiley, 1999: 191-202.

8 Firth-Cozens J, Rayner C. Training in the pre-registration house officer year. Report to North Thames Department of Postgraduate Medicine and Dental Education, 2000

9 Firth-Cozens J. Interventions to improve physicians' well-being and patient care. Soc Sci Med 2001;52:215-22.

\section{Choosing effective strategies for quality improvement}

Since 1998 the UK government has developed a comprehensive strategy for quality improvement for the National Health Service (NHS). This includes national guidelines and standards for clinical care, a national system of inspection and monitoring, and a requirement for all NHS organisations to implement quality improvement strategies. A clinician has been appointed with responsibility for quality improvement within every NHS organisation (the clinical governance lead), but these doctors and nurses have been given little specific guidance on what actually to do. The choice of quality improvement strategies is largely for local leaders to decide.

What does the literature suggest is most likely to be effective? On the whole, single interventions have relatively little effect. Simply distributing guidelines or educational material rarely changes clinical behaviour. ${ }^{1}$ These may be more successful if combined with audit with feedback, computerised prompts, or academic detailing. ${ }^{2-5}$ Financial incentives can produce change,${ }^{6}$ but are a blunt instrument and risk producing perverse incentives. Multiple interventions are generally more effective than single ones. ${ }^{7}$ Substantial claims are made for continuous quality improvement or total quality management strategies, ${ }^{89}$ but there is little clarity about the circumstances in which they produce major change. ${ }^{10}{ }^{11}$ Information on quality of care which is released to the public is mistrusted by doctors, but making information available may stimulate provider organisations to change care. ${ }^{12}$

Given an imperative from the UK government to do something, and a certain amount of guidance from past research on what is likely to work, we now have information from both primary and secondary care on what is actually being done. In this issue of Quality in Health Care Wallace and colleagues report the results of a survey of 86 hospital trusts. ${ }^{13}$ Virtually all had implemented educational programmes, had developed local protocols or guidelines for care, and had established local quality improvement groups. These were followed closely by formal or informal assessment of care by peers. Feedback of performance data was being used in just over half. Campbell et $a l^{14}$ have reported the results of a comparable survey in primary care. Educational activities were again the most commonly reported and in over half the primary care groups surveyed, the whole primary care group (approximately 50 doctors) was closing for one afternoon a month for joint educational activity - a major cultural change for UK general practitioners. Joint audits across practices were common, with half of the groups surveyed feeding back or planning to feed back identifiable comparative information. Unlike the survey of hospital trusts, more than half were providing financial incentives linked to quality improvement.

Wallace et al asked their respondents whether these strategies are likely to be effective. Despite the effort put into educational programmes and guideline development, fewer than half the respondents perceived these to be effective approaches-a view supported by the literature. Creating clinical groups to focus on specific issues was regarded as the most effective of the techniques being used. Clinical governance leads are faced with a paradox. They are under strong pressure to demonstrate activity. Yet some of the things which the literature shows to be most effective agents for change-such as academic detailing-are also heavily resource intensive. Both surveys indicate that modern approaches to behavioural change are being used, with active involvement of clinicians high on the list; in Campbell's survey over $90 \%$ were encouraging the development of personal learning plans by general practitioners. What is clear from both surveys is that a range of simultaneous techniques are being used, which is consistent with the literature on multifaceted interventions being more effective than single ones. This was certainly the experience of one recent quality improvement initiative in UK primary care where major changes in behaviour appeared to have been brought about by a combination of clear leadership and a range of financial and professional incentives. ${ }^{15}$

So what can be made of progress so far? There is no doubt that much activity has taken place. This is perhaps not surprising since a legal "duty for quality" has been put on NHS organisations, and chief executives of NHS trusts can probably expect to lose their jobs where serious deficiencies of quality are found. A wide range of quality improvement activities are now reported, many of which are at least compatible with the literature on interventions likely to produce change. We do not know how the activities reported by clinical governance leads reflect actual change on the ground, or even whether the respondents have the ability to know if such change is taking place-development of IT systems to monitor quality are a high priority across the NHS, but currently they are woefully inadequate. Likewise, we do not know whether those clinicians who have volunteered for roles as clinical governance leads have the experience of organisational change that is needed for them to be effective. In response to this potential deficit, the NHS has started a development programme for quality improvement leaders.

In terms of top down strategy, the UK NHS probably has the most ambitious quality improvement strategy in the developed world. Local leaders have been appointed throughout the NHS with freedom to develop a range of quality improvement programmes, though with no option to do nothing. In terms of actual results it is too early to tell whether the strategy is being successful. Activities reported so far are limited by the resources available, and clinical governance leads identify lack of time and resource as the major barriers to progress. The success of the policy is likely to depend on whether there is continued investment in an 
infrastructure for quality improvement and provision of protected time for quality improvement activities. These will be necessary to produce the cultural change among clinicians that will be necessary if quality improvement is to become a mainstream part of clinical practice, and not just an "add on". ${ }^{16}$

M ROLAND

Director, National Primary Care Research and Development Centre, University of Manchester,

Manchester M13 9PL, UK

mroland@fs1.cpcr.man.ac.uk

1 Freemantle N, Harvey EL, Wolf F, et al. Printed educational materials: effects on professional practice and health care outcomes (Cochrane Review). In: The Cochrane Library 1, Oxford: Update Software, 2001.

2 Thomson O'Brien MA, Oxman AD, Davis DA, et al. Audit and feedback: effects on professional practice and health care outcomes (Cochrane Review). In: The Cochrane Library 1, Oxford: Update Software, 2001.

3 Thomson O'Brien MA, Oxman AD, Davis DA, et al. Audit and feedback versus alternative strategies: effects on professional practice and health care outcomes (Cochrane Review). In: The Cochrane Library 1, Oxford: Update Software, 2001 .

4 Walton RT, Harvey E, Dovey S, et al. Computerised advice on drug dosage to improve prescribing practice (Cochrane Review). In: The Cochrane to improve prescribing practice (Cochra

5 Thomson O'Brien MA, Oxman AD, Davis DA, et al. Educational outreach visits: effects on professional practice and health care outcomes (Cochrane Review). In: The Cochrane Library 1, Oxford: Update Software, 2001.
6 Giuffrida A, Gosden T, Forland F, et al. Target payments in primary care: effects on professional practice and health care outcomes (Cochrane Review). In: The Cochrane Library 1, Oxford: Update Software, 2001

7 Hulscher MEJL, Wensing M, van der Weijden T, et al. Interventions to implement prevention in primary care (Cochrane Review). In: The Cochrane Library 1, Oxford: Update Software, 2001.

8 Berwick DM, Enthoven A, Bunker JP. Quality management in the NHS: the doctor's role: 1. BMf 1992;304:235-9.

9 Berwick DM, Enthoven A, Bunker JP. Quality management in the NHS: the doctor's role: 2. BMf 1992;304:304-8.

10 Shortell SM, Bennett CL, Byck GR. Assessing the impact of continuous quality improvement on clinical practice: what will it take to accelerate progress? Milbank Quarterly 1998;76:593-624.

11 Blumenthal D, Kilo CM. A report card on continuous quality improvement. Milbank Quarterly 1998;76:625-48.

12 Marshall M, Shekelle PG, Leatherman S, et al. Public release of performance data. What do we expect to gain? A review of the evidence. fAMA 2000;283:1866-74.

13 Wallace LM, Freeman T, Latham L, et al. Organisational strategies for changing clinical practice: how trusts are meeting the challenges of clinical governance. Quality in Health Care 2001;10:76-82.

14 Campbell S, Roland M, Wilkin D. Primary care groups and trusts in the new NHS: improving quality of care through clinical governance. BMF 2001 (in press).

15 Spooner A, Chapple A, Roland M. What makes doctors take part a major quality improvement initiative? A qualitative study of the reasons for professional behaviour change. F Health Serv Res Policy 2001 (in press).

16 Davies HTO, Nutley SM, Mannion R. Organisational culture and quality of health care. Quality in Health Care 2000;9:111-9.

\section{Accountability and quality improvement: the role of report cards}

Ensuring accountability and improving quality are two of the most significant challenges facing health systems around the world. The public release of comparative standardised information on quality in the form of "report cards" represents one suggested solution to these complex problems. ${ }^{1}$ Report cards are not new-Florence Nightingale produced a report comparing the mortality rates of London teaching hospitals in $1863^{2}$-but nevertheless they have been embraced with great enthusiasm in many developed countries in recent years. In the UK, for example, the introduction of report cards is one of the specific recommendations in a plan to modernise the National Health Service. ${ }^{3}$

Given this enthusiasm, it is perhaps surprising that we know so little about the uses, benefits, and risks of publicising comparative information. Most of the experience and evidence in this field comes from the USA where report cards have been a prominent feature for the last 15 years. Two papers in this issue of $Q H C$ describe the current state of play in the USA and help to develop our understanding of whether and how report cards fulfil the ambitious claims made of them.

It may seem self-evident that the general public should be the primary audience for the "public" release of comparative information. Not so, it would appear, according to Schneider and Lieberman. ${ }^{4}$ Even in the consumer orientated USA where users have expressed a desire for the information in principle, ${ }^{5}$ in practice they do not appear to search for, understand, trust, or make use of the data. This must have come as something of a shock to the proponents of report cards who expected consumers to respond to the information in a rational way, weighing up the costs and benefits, making a judgement about which providers were best, and driving low quality providers out of the competitive market.
Researchers and policy makers have attempted to explain this apparent paradox in terms of deficiencies in the content, presentation, or dissemination of the data or in terms of the lack of real choice for many US citizens. In doing so they may have missed the point. The apparent disconnection between the public's demand for information and their use of report cards is perhaps not as paradoxical as it first appears. It is possible that service uses are simply saying that they want the information to be available and that they are dissatisfied with what they perceive as the veil of secrecy and professional protectionism currently seen in health care. It is, of course, possible that better data or well informed and empowered consumers will be more willing to use report cards in the future, but it is probable that the impact of report cards on consumer behaviour will always be marginal. Perhaps the rational choice model of decision making, so admired by economists, is an inappropriate way of explaining the public's choice of healthcare providers. Is it possible that the public simply does not want to behave in a consumerist way in all aspects of modern life? Sociobehavioural models of decision making ${ }^{6}$ which recognise the complex input of beliefs, experiences, enabling factors, and the unique self-perception of problems are more useful in explaining the public response to report cards. In an attempt to understand the role of report cards it might then be more productive to re-focus attention on the mechanisms of lay decision making, the merits of expert held knowledge, and the role of advocates in making use of complex comparative information.

Those who are disappointed by the apparent disinterest shown by consumers may gain solace from the second paper on public disclosure in this issue. Davies suggests that US hospitals do make use of comparative information and that the public release of the data acts as a catalyst by reminding, refocusing, or shaming the organisations into 
giving priority to quality improvement. ${ }^{7}$ Again, the author suggests that the data are not used in an entirely rational way. Respondents tended to use the report cards to confirm their views about the performance of their own and other organisations, views which were based primarily on informal contacts and personal experiences. If the "hard" data did not support their prejudice, then they were more likely to judge the data to be incorrect than to accept that their own views might be wrong.

Nevertheless, the report cards served an important purpose by stimulating the organisations to look beyond the published data and encouraging them to develop and improve their own internal data systems. This suggests that the data contained in report cards have to be accurate enough to engage the attention of those whose responsibility it is to take action, but does not have to be perfect. If correct, this has important policy implications. The New York Cardiac Surgery Report System, for example, disseminated sophisticated risk adjusted data which enabled reasonably valid and reliable judgements to be drawn about the relative performance of individual cardiac surgeons and hospitals in the state of New York. ${ }^{8}$ Such data are extremely costly and time consuming to produce. This might be a necessary expense if the aim of the data is to make proscriptive judgements about fitness to practice, but may not be required if the aim of the report cards is to encourage engagement with the quality improvement process.

The introduction of report cards in the USA is not a shining example of implementing a radical and innovative health policy. The enthusiasm for the public reporting of performance is understandable; it must be right to provide information in an open and democratic society and it must be better for all stakeholders to be informed than to be kept in the dark. However, the initial expectations of report cards in the USA seem in retrospect to be naïve. There can be little doubt that comparative information about quality of care will be freely available in most developed countries within the next decade. Those who are responsible for introducing report cards and those who wish to make use of them would do well to examine the rapidly expanding literature in this field.

M N MARSHALL

Professor of General Practice,

National Primary Care Research and Development Centre,

University of Manchester,

Manchester M13 9PL, UK

martin.marshall@man.ac.uk

1 Marshall MN, Shekelle PG, Leatherman S, et al. Dying to know: public release of comparative data in health care. London: Nuffield Trust, 2000.

2 Nightingale F. Notes on hospitals. 3rd edn. London: Longman, Green, Longman, Roberts and Green, 1863 .

3 Department of Health. The NHS plan: a plan for investment, a plan for reform. London: Department of Health, 2000

4 Schneider EC, Lieberman T. Publicly disclosed information about the quality of health care: response of the US public. Quality in Health Care 2001; 10:96-103.

5 Edgman-Levitan S, Cleary P. What information do consumers want and need? Health Affairs 1996;15:42-56.

6 Rogers A, Hassell K, Nicolaas G. Demanding patients? Analysing the use of primary care. Buckingham, UK: Open University Press, 1999.

7 Davies HTO. Public release of performance data and quality improvement: internal responses to external data by US health care providers. Quality in Health Care 2001;10:104-10.

8 Chassin M, Hannan E, DeBuono B. Benefits and hazards of reporting medical outcomes publicly. $N$ Engl f Med 1996;334:394-8.

\section{Erring is human: will we cross the quality chasm?}

A local rural land agent with some newly acquired knowledge was eagerly entreating a farmer to quadruple his crop yields by adopting new methods. After an exhortation of some length, the farmer raised his hand to silence the fellow and drawled, "Sorry, not interested. I already know how to be a much better farmer than I am today". The research of the late Nobel Laureate Herbert Simon, who studied decision making processes in economic organisations, revealed that humans have a "bounded rationality" that "satisfices" rather than constantly seeks to "optimise". As a matter of human nature and disposition, human organisations do not constantly seek to be better and do better. Instead, it is quite easy to find clear evidence of underperformance in organisations relating to every field of human endeavour. Like other organisations, health care institutions don't improve dramatically simply by pointing out lapses in their performance.

For the past 30 years the Institute of Medicine (IOM) in Washington has published many health policy reports that reflect the thoughtful expert advice of its multidisciplinary committees - reports that outline steps capable of generating better health care. ${ }^{1}$ Since 1996 the IOM has sought explicitly to influence the quality of US health care over the next decade. A committee formed in 1998 to recommend the action needed to generate lasting and continual threshold improvement in the US health system has now produced two reports, To err is human: building a safer health system and Crossing the quality chasm: a new health system for the 21st century. ${ }^{2}{ }^{3}$ Both reports are receiving a great deal of media, professional, and government attention. But these are still early days and, while the impact of these reports on the quality and safety of health care has yet to be realised, Professor Kenneth Shine, the IOM President, has noted that the first report has taken on a life of its own.

The first report focuses on the substantial body of research that reveals major shortcomings in both the safety and quality of health care. For example, 44 000-98 000 Americans are estimated to die unnecessarily each year in hospital and half of these deaths are considered potentially avoidable. ${ }^{2}$ Similar quality problems do not typify all US industries - for example, during the calendar year 2000 there was not a single fatality from commercial airline use. Health care clearly has a great deal to learn. Furthermore, unless we change our ways soon and develop systems of care which are safe and capable of delivering good quality care, the demographic impact of an aging population with rising chronic care needs will add further stress to a system which cannot now always guarantee safety, let alone continuous improvement. Even new tools such as information technology and an enormously expanded knowledge base of health care will have far less effectiveness unless the system as a whole is changed, made safer, and is capable of consistently delivering good quality care.

The second report, published last month, calls for a national effort to create a system that is fully committed to being safe, effective, patient centred, timely, efficient, and equitable. A health care system worthy of the name must commit "to continually reduce the burden of illness, injury, and disability, and to improve the health and functioning of the people ..." To deliver such dramatic changes will require leadership, broad commitment, and the intelligent deployment of resources. 
Clearly, a baseline requirement for dramatic improvements in health care is a robust national health information infrastructure. The UK's Information for health strategy has its parallel in the vision for a national health information infrastructure described by the National Committee on Vital and Health Statistics. ${ }^{4}$ Neither is getting the funding it deserves and substantial financing at the national level will be required. Furthermore, while technology is a crucial part of the solution, the whole process of reform must centre on the patient and the facilitation of the healing relationships between patients and healthcare workers. The system must allow patients to make informed choices and, as far as is feasible, to control their care.

Setting dramatically high aspirations for safety and quality in individual care and system performance is certain to generate a great deal of interest and action. Or will it? And what impact, if any, will these reports from the USA have in Europe? Most experts familiar with the health systems of economically developed economies see many parallels and similarities despite national idiosyncrasies. While the findings and recommendations of these reports appear to be generalisable, the real interest is to see how much action will result and, if reforms are implemented, to ask whether the process of implementation is generalisable.

In the USA early public and professional response to the safety report was generally quite supportive. However, there are clinicians who believe that the report was unfair and misguided and that patients' confidence in doctors and hospitals will be seriously undermined by these reports from the IOM. So far, there has been nothing to suggest negative public reaction despite evidence that half of the population has been following the reports in the media. Equally, there is little evidence that hospitals are any safer for patients. Having said that, there has been a generally salutary response to the report throughout the USA and some action at the level of the federal government has occurred as well. Millions of new dollars are being directed to produce regular national reports on the quality of care and to increase the support for research on quality and incident reporting.

Some wag noted that there is nothing like a "near death" experience to focus one's attention sufficiently to change one's errant ways. Whether the constant drip feed of news articles describing a graphic example of poor quality care qualifies either the US system or the NHS in the UK as "near death" or not, I leave to others to decide. However, the IOM reports do offer a fairly detailed prescription for responsible leadership from the health professions. Certainly, nations do need an industrial strength health care system worthy of the name. Admittedly, media interest, political scrutiny, layers of complex and conflicting regulations, and dense professional cultures make health systems notoriously difficult to change, even when they wish to do so. But the evidence that supports the need for change is there.

Whether that farmer changed his farming practice was ultimately up to him, but that didn't mean that the consequences of his decision wouldn't affect his crops, his output. Apparently, he was sufficiently satisfied to continue "satisficing". We, in health care, have our own food chain to worry about and its output is the health of both our patients and the population. We now know that the farmer - that is, the individual care worker - is only as good as the system around him. Totally independent practitioners working their own little plots just won't get the result that statistics show is delivered by good systems. The difficulty for healthcare professionals who learn to be good individual practitioners is that today's health care is dependent on the good individual practitioner working well in an interprofessional team within a good, well thought out health care system. If individual doctors, nurses, managers, and governments understand this, realise that we need urgently to reform, and offer transformational leadership, historic improvements in our care systems can result within a decade. It is true that the details for accomplishing these dramatic results are not all worked out. But surely our patients will do better if, in the future, we "satisfice" within a system enhanced with computer based decision support and embedded safety protocols. Surely this is our hour to do this essential work. Let's get on with it. Remember, our role as health professionals is to help while doing no harm. Unnecessary deaths are simply not acceptable.

Professor Detmer was a member of the committee of both reports.

\section{E DETMER}

Gillings Professor of Health Management,

University of Cambridge,

Cambridge CB2 1Af, UK

d.detmer@jims.cam.ac.uk

1 Institute of Medicine. www.iom.edu (accessed 12 April 2001).

Insititute of Medicine. To err is human: building a safer health system. Washington: National Academy Press, 2000.

3 Institute of Medicine. Crossing the quality chasm: a new health system for the 21st century. Washington: National Academy Press, 2001

4 NHS Executive. Information for health. London: NHS Executive, 2000, www.doh.gov.uk/nhsexipu/strategy/index.htm (accessed 12 April 2001) 5 National Committee on Vital and Health Statistics. Toward a national health information infrastructure. Washington: National Committee on Vital and 12 April 2001). 